Sibiry Albert KABORÉ $\hat{E}^{1,2}$

Brigitte BASTIDE ${ }^{2}$

Salifou TRAORÉ ${ }^{1}$

Joseph Issaka Boussım ${ }^{1}$

${ }^{1}$ Université de Ouagadougou

Unité de formation et de recherche

en sciences de la vie et de la terre

Laboratoire de biologie et écologie végétales

03 BP 7021, Ouagadougou 03

Burkina Faso

2 Institut de l'environnement

et de recherches agricoles

Département productions forestières

03 BP 7047, Ouagadougou 03

Burkina Faso

\section{Dynamique du karité, Vitellaria paradoxa,
dans les systèmes agraires
du Burkina Faso Vitellaria paradoxa,
dans les systèmes agraires
du Burkina Faso Vitellaria paradoxa,
dans les systèmes agraires
du Burkina Faso}

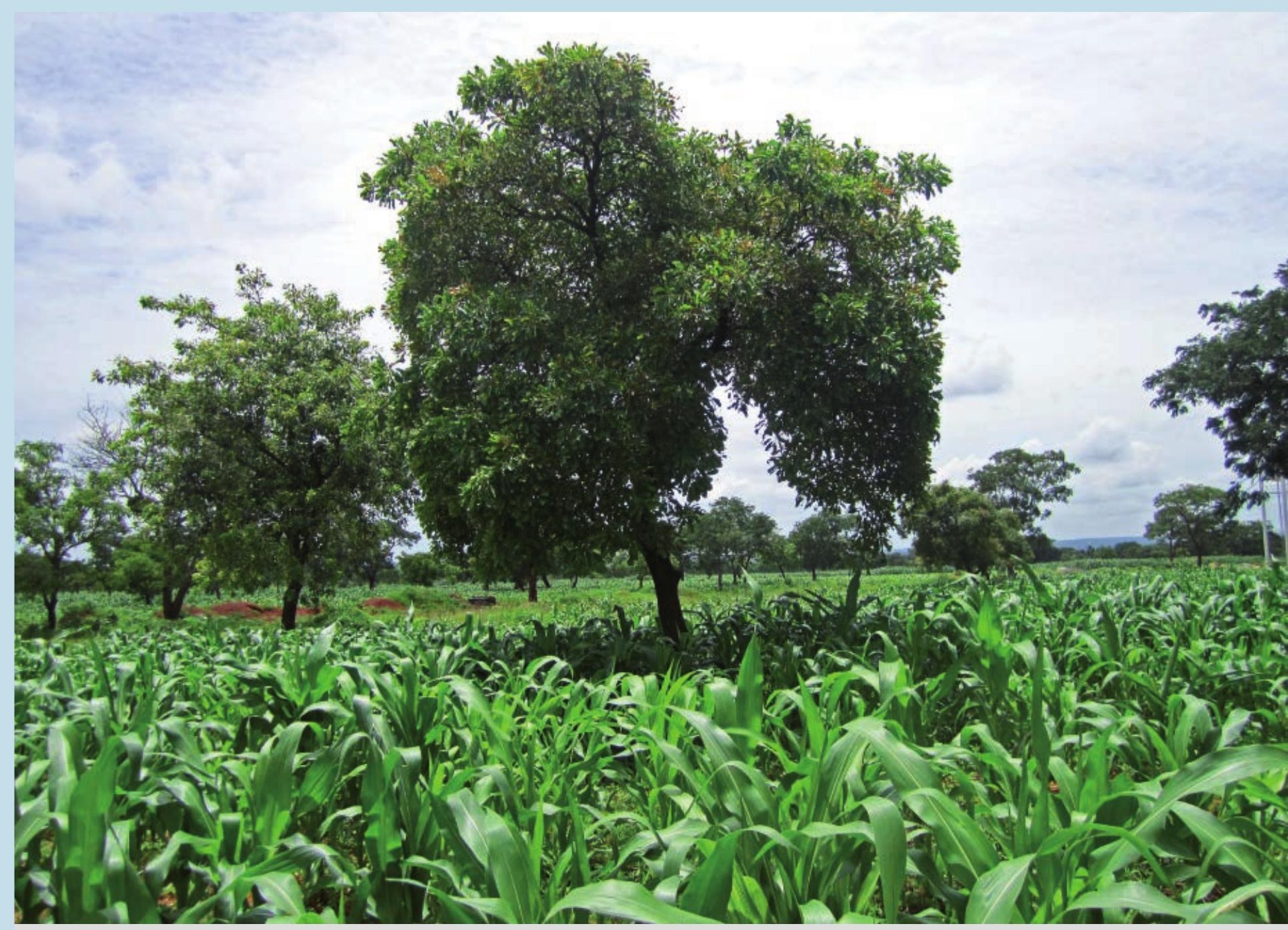

Photo 1.

Karité dans un champ de maïs.

Photo S. A. Kaboré. 


\section{RÉSUMÉ}

DYNAMIQUE DU KARITÉ, VITELLARIA PARADOXA, DANS LES SYSTĖMES AGRAIRES DU BURKINA FASO

L'article présente les résultats d'une étude conduite dans la zone sud-soudanienne du Burkina Faso, axée sur les pratiques agricoles paysannes. II s'agit, en particulier, de l'impact exercé par la diminution de la durée de mise en jachère et par l'augmentation de la période d'exploitation des champs sur la dynamique des populations du karité, Vitellaria paradoxa. Afin de contribuer à la gestion durable de l'espèce, un inventaire exhaustif des karités a été mené dans des champs et des jachères de différents âges, au moyen d'un dispositif d'échantillonnage imbriqué. En outre, une enquête a été effectuée auprès des propriétaires de différentes parcelles pour mieux cerner les pratiques influençant la dynamique des karités. Les résultats montrent que les densités des karités adultes chutent notablement au sein des champs de plus de quinze ans. À l'inverse, les densités s'accroissent nettement avec l'âge des jachères. Les paysans n'épargnent pas les plantules lors des opérations culturales, ainsi le renouvellement des karités n'est-il guère assuré en champ et la dynamique de l'espèce y est donc régressive. En revanche, une abondante régénération s'installe quand la parcelle est laissée en jachère. Si le contexte écologique le permet, la pratique des longues jachères d'au moins quinze années est une bonne technique pour restaurer les parcs à karités. Dans les zones où de telles jachères sont impossibles à mettre en place, il est fortement conseillé aux producteurs de préserver quelques plantules dans leurs champs et de les entretenir afin d'amorcer le rajeunissement des parcs.

Mots-clés : Vitellaria paradoxa, dynamique, régénération, gestion durable des parcs, pratiques culturales, Burkina Faso.

\section{ABSTRACT}

\section{DYNAMICS OF THE SHEA TREE, VITELLARIA PARADOXA, IN AGRARIAN SYSTEMS IN BURKINA FASO}

This article describes the results of a study on peasant farming practice in Burkina Faso's southern Sudanian zone, focusing in particular on the impact of shorter fallow periods and longer cultivation periods on the dynamics of the shea tree species Vitellaria paradoxa. To contribute to sustainable management of the species, an exhaustive inventory of shea trees was made in fields and fallow lands of different ages, using a nested sampling technique. A further survey was conducted among owners of different plots to help identify practices that influence shea tree dynamics. Our results show that the density of adult shea trees lessens notably in fields cultivated for more than 15 years. Conversely, their density clearly increases with the age of fallow lands. Farmers weed out shea seedlings when tilling their fields, so that the trees do not regenerate in cultivated lands and are therefore in decline. However, they regenerate abundantly when the plot is left fallow. In a favourable ecological context, long fallow periods of at least fifteen years are a sound technique for restoring shea tree parkland. In zones where long fallow periods are not possible, farmers are strongly advised to maintain a few young seedlings in their fields to help regenerate shea tree parklands.

Keywords: Vitellaria paradoxa, dynamics, regeneration, sustainable parkland management, cultivation practice, Burkina Faso.

\section{RESUMEN}

\section{DINÁMICA DEL KARITÉ, VITELLARIA PARADOXA, EN LOS SISTEMAS AGRARIOS DE BURKINA FASO}

El artículo presenta los resultados de un estudio realizado en la zona climática sursudanesa de Burkina Faso y centrado en las prácticas agrícolas campesinas. Se aborda, en particular, el impacto ejercido por la reducción del tiempo de barbecho y por el aumento del período de aprovechamiento de los campos en la dinámica de las poblaciones de karité, Vitellaria paradoxa. Para contribuir al manejo sostenible de la especie, se efectuó un inventario exhaustivo de árboles de karité en campos y barbechos de diferentes edades mediante un diseño de muestreo anidado. Además, se realizó una encuesta entre los propietarios de las distintas parcelas para definir mejor las prácticas que influyen en la dinámica del karité. Los resultados muestran que las densidades de los árboles adultos disminuyen considerablemente en los campos de más de quince años. Por el contrario, las densidades se incrementan de forma significativa con la edad de los barbechos. Los agricultores no respetan los plantones durante las operaciones de cultivo, por lo que la renovación de los árboles en el campo prácticamente no se produce y la dinámica de la especie es, por tanto, regresiva. Sin embargo, se produce una abundante regeneración cuando se deja la parcela en barbecho. Si el contexto ecológico lo permite, la práctica de barbechos largos de, al menos, quince años es una buena técnica para restaurar el arbolado de karité. En aquellas zonas en las que resulte imposible establecer estos barbechos, se recomienda firmemente a los productores que salvaguarden algunos plantones en sus campos y que las mantengan para dar inicio el rejuvenecimiento de los parques.

Palabras clave: Vitellaria paradoxa, dinámica, regeneración, manejo sostenible de parques, prácticas de cultivo, Burkina Faso. 


\section{Introduction}

En Afrique soudanienne, l'homme a toujours entretenu des rapports étroits avec les espèces arborées des parcs agroforestiers. Parmi ces espèces figure le karité, Vitellaria paradoxa, qui est l'une des espèces dominantes dans les systèmes agraires soudano-sahéliens du Burkina Faso. Préservés dans les champs où ils bénéficient des labours et de la protection contre le feu, les arbres deviennent plus vigoureux et plus productifs que les sujets des formations naturelles (Bonkoungou, 1987 ; Kelly et al., 2007) (photo 1). Cependant, la durabilité des ressources des parcs arborés déjà vieillissants est remise en question par l'augmentation de la pression démographique et par les modes de gestion agricole et pastorale (BONKoungou, 1987 ; PELTIER et al., 2007). Dans un contexte de pression anthropique sans cesse croissante sur les terres, il est possible d'observer un raccourcissement de la durée de mise en jachère et une exploitation plus prolongée des champs (Augusseau et al., 2006 ; ASSÉ, LASSOIE, 2011). Or certains auteurs, parmi lesquels SERPEnTIÉ et al. (1996), ont démontré le rôle bénéfique des jachères dans la dynamique du karité.

Les produits issus de cette espèce, notamment le beurre de karité, occupent une place très importante dans la vie des ménages des zones rurales et dans l'économie de nombreux pays (HALL et al., 1996 ; SANOU et al., 2004 ; Nouvellet et al., 2006 ; GWALI et al., 2012). Les amandes, issues des graines récoltées par les femmes, sont transformées en beurre ou vendues séchées (photos 2 à 4). Entre 1999 et 2006, le Burkina Faso a exporté des graines de karité d'une valeur de plus de treize milliards de francs $\mathrm{Cfa}^{1}$ (INSD, 2009). La production fruitière est irrégulière si bien que les prix des amandes fluctuent d'une année à l'autre selon l'offre et la demande. Cependant, malgré la diversité des utilisations du beurre de karité dans la cosmétique, la chocolaterie et les soins de santé, l'espèce ne fait pas l'objet de plantations (HALL et al., 1996). Actuellement, seul le processus de la régénération naturelle assure le renouvellement de ses pieds (Augusseau et al., 2006). La filière karité fait donc face aux difficultés de maintien et de croissance de la production du fait de la baisse de la fréquence des arbres sur le terrain. En effet, Chevalier (1946) a estimé la densité des karités à 230 arbres par hectare dans les années 1940 dans la zone soudanienne ; mais cette densité a chuté et se situe actuellement aux environs de onze arbres à l'hectare en moyenne (NIKIÉMA et al., 2003). C'est pourquoi il devient urgent de s’intéresser à la dynamique des populations de cette espèce à haute valeur économique au sein de l'espace agraire, afin d'améliorer sa gestion in situ et d'évaluer l'impact des pratiques de gestion et d'occupation des terres sur la structure de ses peuplements naturels.

${ }^{1} 1 €=656$ francs Cfa ; 1 \$US $=524$ francs Cfa.
L'élagage, qui consiste à couper les branches pour éliminer les phanérogames parasites (notamment ceux des genres Agelanthus et Tapinanthus) et pour rajeunir les vieux pieds pour une meilleure production fruitière (BAYALA et al., 2008 ; GWALI et al., 2012), et le cernage par incision du tronc afin d'augmenter le rendement en fruits (LAMIEN, 2006) sont des techniques pouvant améliorer les parcs à karités. Toutefois, ces techniques exigent des karités adultes qui se raréfient dans plusieurs régions. Le greffage avec des greffons issus des meilleurs individus de karité (SANOU et al., 2004 ; RABBILD et al., 2011) et la production de plants par marcottage (RABBILD et al., 2011) donnent des résultats encourageants mais difficilement applicables par les paysans ; c'est pourquoi un accent particulier doit être mis sur la régénération naturelle. En considérant l'hypothèse selon laquelle les types d'utilisation des terres influencent la dynamique du karité, il s'agit de répondre à la question suivante : «Quel est l'impact sur la dynamique du karité des pratiques agricoles paysannes qui consistent, en particulier, à diminuer la durée de mise en jachère et à augmenter la période d'exploitation des champs ? „ Cet article tente d'y répondre à partir d'une étude réalisée dans les systèmes agraires du terroir de Sobaka (zone sud-soudanienne du Burkina Faso) tels qu'ils sont gérés actuellement par les paysans.

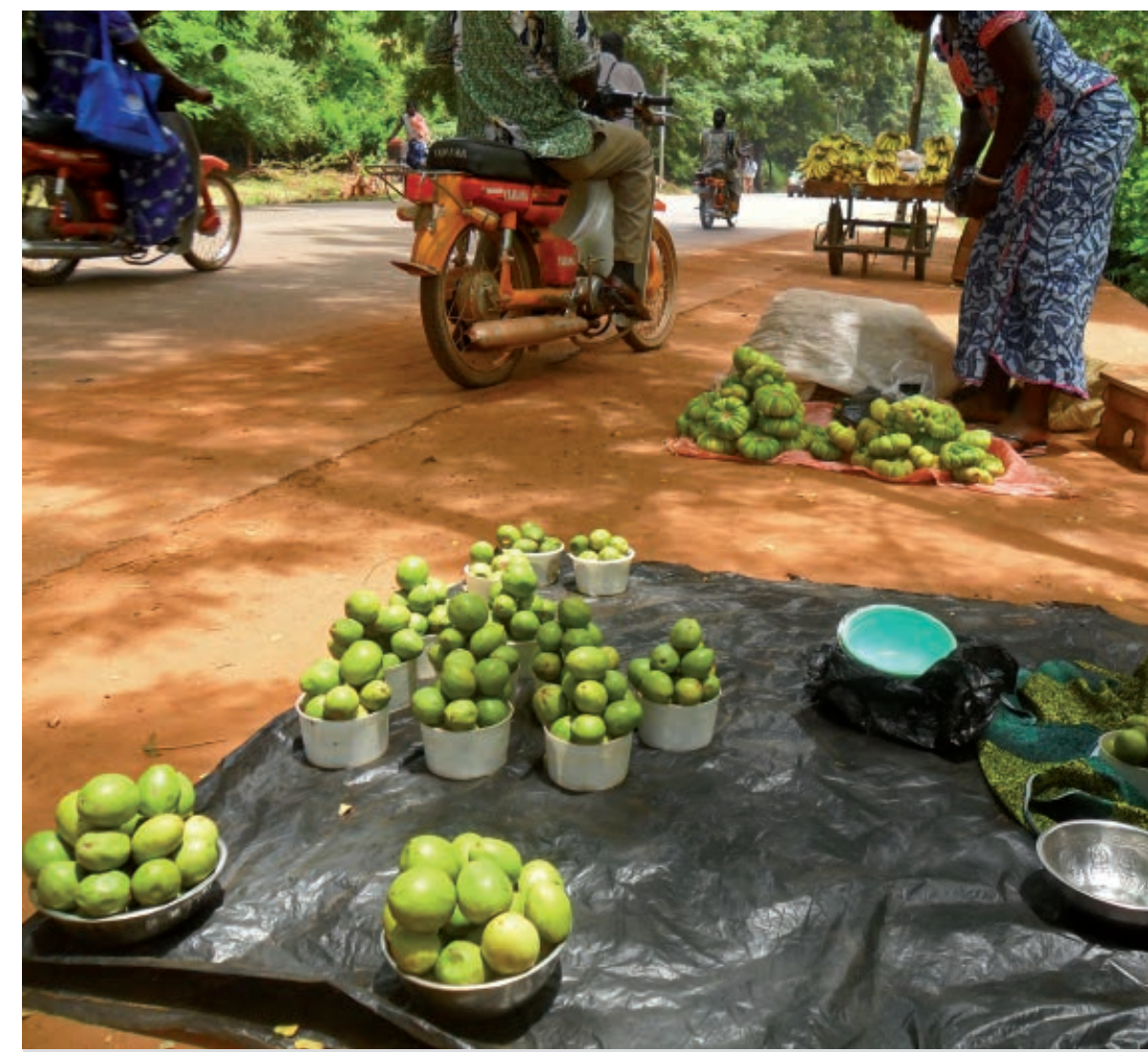

Photo 2.

Fruits de karité vendus au bord d'une avenue de Ouagadougou : de l'avant vers l'arrière-plan, les tas sont à 500, 200 et 100 francs Cfa.

Photo S. A. Kaboré. 


\section{Matériel et méthodes}

\section{Milieu d'étude}

Le terroir de Sobaka, en zone sud-soudanienne du Burkina Faso, est situé à proximité de la forêt du Nazinon, à $70 \mathrm{~km}$ au Sud de Ouagadougou, dans la zone centrale de l'aire de distribution du karité au Burkina Faso. II s'y trouve un important peuplement de karités. Le sorgho et le mil constituent les principales cultures. Les modes d'utilisation des terres y sont très diversifiés et représentatifs du contexte écologique de la zone soudanienne. Il faut distinguer les champs de case et les champs de brousse. Les premiers ne sont jamais mis en jachère. Par contre, lorsque le paysan constate un appauvrissement des parcelles, il laisse certains champs de brousse en jachère pendant un certain temps avant une nouvelle remise en jachère (SomÉ, 1996).

Le contexte climatique est caractérisé par une saison pluvieuse de 5 à 6 mois avec une pluviosité annuelle variant entre 750 et 1050 mm (période 1995-2009) et une saison sèche de 6 à 7 mois. L'humidité relative moyenne de l'air, de $85 \%$ en août, descend progressivement à $35 \%$ en janvier. Mars et avril sont les mois les plus chauds de l'année (température moyenne maximale : $40^{\circ} \mathrm{C}$ ), décembre et janvier les plus frais (température moyenne minimale : $17^{\circ} \mathrm{C}$ ). Les sols ferrugineux tropicaux lessivés sur cuirasse et carapace sont dominants (SomÉ, 1996). Le karité est présent sur une gamme variée de types de sols. Il est peu abondant au bord des cours d'eau.

\section{Échantillonnage}

Le dispositif d'échantillonnage est un plan imbriqué à deux facteurs considérant les modes d'utilisation des terres (jachère, champ) et leur durée. Une enquête auprès des paysans a permis de recenser six grands types de peuplements répartis comme suit :

- jeunes champs, exploitation depuis 2 ans ;

- champs d'âge intermédiaire, exploitation depuis 6 ans ;

- vieux champs, exploitation depuis plus de 15 ans;

- jeunes jachères, abandon depuis 2 à 3 ans ;

- jachères d'âge intermédiaire, abandon depuis 6 à 8 ans ; - vieilles jachères, abandon depuis plus de 15 ans.
Un tirage aléatoire a permis de retenir trois parcelles dans chaque type de peuplement parmi ceux recensés auprès de la population. Sur chacune des parcelles retenues, une placette carrée de 50 m de côté (2500 m²) a été délimitée, au sein de laquelle les relevés ont été effectués.

\section{Collecte et traitement des données}

\section{Étude de la structure des populations de Vitellaria paradoxa}

L'inventaire de la population de karités a été conduit en décembre 2008. Il a consisté à recenser tous les pieds de l'espèce et à mesurer les hauteurs, les circonférences à la base des troncs et à 1,30 m du sol, les circonférences des troncs à la base des houppiers, les diamètres Est-Ouest et Nord-Sud des houppiers et les hauteurs des troncs. Tout individu ayant moins d'un mètre de hauteur est considéré faire partie de la régénération. Les coordonnées Gps de tous les sujets, adultes comme plantules, ont été relevées. Les autres ligneux ont été aussi inventoriés.

\section{Enquête sur les pratiques agricoles}

Une enquête a été conduite en 2009 auprès des propriétaires des différentes parcelles échantillonnées. Dix-huit propriétaires de champs et/ou de jachères ont été interrogés afin de cerner les pratiques culturales qui pourront influer sur la dynamique du karité. Le questionnaire s'est focalisé sur certaines informations relatives à la pratique de l'alternance entre champ et jachère, à la protection des plantules de Vitellaria paradoxa dans les champs, aux modes de gestion du karité et aux pratiques agricoles.

\section{Traitement et analyse des données}

Des classes de hauteur ont été définies pour l'élaboration de la structure verticale de la population. La structure spatiale des différents pieds a été réalisée à l'aide de leurs coordonnées Gps. Les densités des karités ont été calculées dans les différentes unités. Une analyse de variance (Anova) a été effectuée. Le test LSD de Fischer a été utilisé avec un seuil de risque de $5 \%$. Les résultats fournis par l'Anova permettent d'évaluer la qualité de l'ajustement du modèle aux données. Ces analyses ont été réalisées à l'aide du logiciel XLStat Pro version 7.5.2.

Tableau I.

Valeurs moyennes des mesures sur la population de Vitellaria paradoxa à Sobaka, Burkina Faso.

\begin{tabular}{|c|c|c|c|c|c|c|c|c|c|}
\hline \multirow[b]{3}{*}{ Jeunes champs } & \multicolumn{7}{|c|}{ Karités de $1 \mathrm{~m}$ et plus de hauteur totale } & \multicolumn{2}{|c|}{$\begin{array}{l}\text { Régénération } \\
\text { (moins de } 1 \mathrm{~m} \text { de hauteur) }\end{array}$} \\
\hline & $\begin{array}{l}\text { Circonférence } \\
\text { à } 1,30 \mathrm{~m}(\mathrm{~cm})\end{array}$ & $\begin{array}{l}\text { Circonférence } \\
\text { à la base }(\mathrm{cm})\end{array}$ & $\begin{array}{c}\text { Circonférence } \\
\text { au houppier (cm) }\end{array}$ & $\begin{array}{c}\text { Diamètre E-0 } \\
\text { du houppier (m) }\end{array}$ & $\begin{array}{c}\text { Diamètre N-S } \\
\text { du houppier (m) }\end{array}$ & $\begin{array}{c}\text { Hauteur } \\
\text { du tronc }(m)\end{array}$ & $\begin{array}{l}\text { Hauteur totale } \\
\text { du pied (m) }\end{array}$ & $\begin{array}{c}\text { Hauteur de } \\
\text { l'individu (cm) }\end{array}$ & $\begin{array}{c}\text { Diamètre } \\
\text { à la base }(\mathrm{cm})\end{array}$ \\
\hline & 58,8 & 73,0 & 56,0 & 4,7 & 4,9 & 2,0 & 5,8 & 5,5 & 1,4 \\
\hline $\begin{array}{l}\text { Champs d'âge } \\
\text { intermédiaire }\end{array}$ & 94,1 & 119,0 & 91,5 & 7,7 & 7,7 & 2,3 & 7,7 & 3,1 & 1,0 \\
\hline Vieux champs & 101,8 & 121,9 & 101,6 & 7,8 & 8,2 & 2,2 & 8,1 & 4,8 & 1,3 \\
\hline Jeunes jachères & 82,5 & 100,7 & 77,1 & 6,4 & 6,8 & 1,9 & 6,8 & 7,4 & 1,4 \\
\hline $\begin{array}{l}\text { Jachères d’âge } \\
\text { intermédiaire }\end{array}$ & 75,4 & 95,3 & 67,4 & 6,3 & 6,1 & 2,2 & 6,5 & 6,2 & 1,2 \\
\hline Vieilles jachères & 38,8 & 43,4 & 29,1 & 3,4 & 3,5 & 1,7 & 4,0 & 9,0 & 1,6 \\
\hline
\end{tabular}




\section{Résultats}

\section{Structure des populations de Vitellaria paradoxa}

\section{Caractéristiques dendrométriques des karités}

Le tableau I présente les valeurs moyennes des paramètres dendrométriques des karités dans les différents types de parcelles. Les individus des vieux champs présentent les plus grandes valeurs sauf pour la hauteur du fût. La circonférence moyenne des troncs à 1,30 mètre passe de $102 \mathrm{~cm}$ dans les vieux champs à $39 \mathrm{~cm}$ dans les vieilles jachères. Il est possible de déduire du tableau I que le diamètre moyen du houppier peut atteindre une taille de l'ordre de huit mètres dans les vieux champs ou les champs d'âge intermédiaire et presque cinq mètres dans les jeunes champs. En moyenne, la projection du houppier d'un karité au sol couvre $50 \mathrm{~m}^{2}$ pour les vieux champs, $47 \mathrm{~m}^{2}$ pour les champs d'âge intermédiaire et $18 \mathrm{~m}^{2}$ pour les jeunes champs. En rapportant ces surfaces élémentaires aux différentes densités des karités (tableau II), il s'avère que les karités couvrent $1142 \mathrm{~m}^{2}$ à l'hectare dans les vieux champs, $1678 \mathrm{~m}^{2} /$ ha (17\% de la superficie au sol) dans les champs d'âge intermédiaire et $603 \mathrm{~m}^{2} /$ ha dans les jeunes champs.

\section{Structure verticale des populations}

À Sobaka, les jeunes karités se raréfient avec l'âge des champs (figure 1a). Certes, parmi les karités épargnés lors de l'ouverture des nouveaux champs figurent des jeunes individus de 3 à $5 \mathrm{~m}$ et des individus matures, mais par contre, dans les vieux champs d'une quinzaine d'années exploités en continu sans aucune remise en jachère, ne subsistent que des vieux karités. Quel que soit le type de champ, aucune des plantules retrouvées ne dépasse $10 \mathrm{~cm}$ de hauteur.

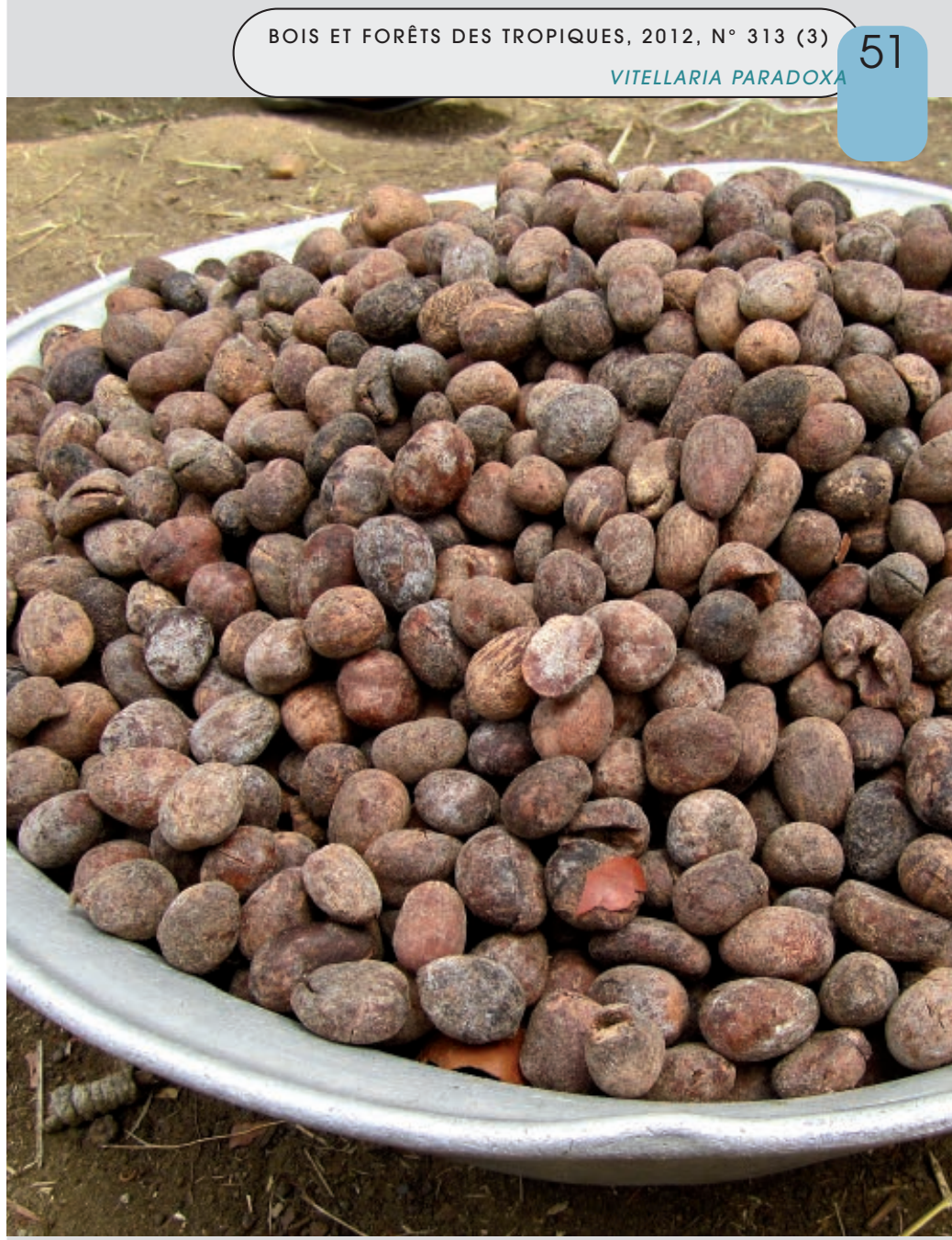

Photo 3.

Amandes de karité en vente dans un marché.

Photo S. A. Kaboré.

Tableau II.

Densités de Vitellaria paradoxa selon les classes de hauteur $(\mathrm{H})$ des individus dans les différents types de parcelles en décembre 2008 à Sobaka.

Types de parcelles

\begin{tabular}{l|c|c|c} 
& & & \\
Vieux champs & $0,0^{\mathrm{b}}$ & $22,7^{\mathrm{b}}$ & $22,7^{\mathrm{b}}$ \\
& $(0,0)$ & $(4,6)$ & $(4,6)$ \\
\hline Champs d'âge intermédiaire & $0,0^{\mathrm{b}}$ & $36,0^{\mathrm{b}}$ & $36,0^{\mathrm{b}}$ \\
& $(0,0)$ & $(8,0)$ & $(8,0)$ \\
\hline Jeunes champs & $2,7^{\mathrm{b}}$ & $30,7^{\mathrm{b}}$ & $33,3^{\mathrm{b}}$ \\
& $(2,3)$ & $(18,9)$ & $(19,7)$ \\
\hline Jeunes jachères & $5,3^{\mathrm{b}}$ & $22,7^{\mathrm{b}}$ & $28,0^{\mathrm{b}}$ \\
& $(11,5)$ & $(4,6)$ & $(11,5)$ \\
\hline Jachères d'âge intermédiaire & $20,0^{\mathrm{b}}$ & $50,0^{\mathrm{ab}}$ & $70,0^{\mathrm{b}}$ \\
& $(0,0)$ & $(42,4)$ & $(42,4)$ \\
\hline Vieilles jachères & $109,3^{\mathrm{a}}$ & $85,3^{\mathrm{a}}$ & $194,7^{\mathrm{a}}$ \\
& $(97,5)$ & $(29,5)$ & $(98,0)$
\end{tabular}

Individus de hauteur $<1 \mathrm{~m}$ (individus/ha)

$\mathrm{H}<0,5 \mathrm{~m} \quad 0,5 \leq \mathrm{H}<1 \mathrm{~m} \quad$ Densité totale $\mathrm{H}<1 \mathrm{~m}$

\begin{tabular}{|c|c|c|}
\hline $\begin{array}{c}212,0^{a} \\
(241,0)\end{array}$ & $\begin{array}{c}0,0^{b} \\
(0,0)\end{array}$ & $\begin{array}{r}212,0^{a} \\
(241,0)\end{array}$ \\
\hline $\begin{array}{r}717,3^{a} \\
(611,8)\end{array}$ & $\begin{array}{c}0,0^{b} \\
(0,0)\end{array}$ & $\begin{array}{r}717,3^{\circ} \\
(611,8)\end{array}$ \\
\hline $\begin{array}{r}361,3^{a} \\
(203,3)\end{array}$ & $\begin{array}{l}1,3^{b} \\
(2,3)\end{array}$ & $\begin{array}{r}362,6^{\mathrm{a}} \\
(204,2)\end{array}$ \\
\hline $\begin{array}{c}1132,0^{a} \\
(1020,7)\end{array}$ & $\begin{array}{c}18,7^{a b} \\
(32,3)\end{array}$ & $\begin{array}{r}1150,7^{a} \\
(1052,6)\end{array}$ \\
\hline $\begin{array}{r}1398,0^{a} \\
(579,8)\end{array}$ & $\begin{array}{c}8,0^{\mathrm{ab}} \\
(11,3)\end{array}$ & $\begin{array}{r}1406,0^{a} \\
(591,1)\end{array}$ \\
\hline $\begin{array}{r}2077,3^{a} \\
(2526,4)\end{array}$ & $\begin{array}{c}44,0^{a} \\
(44,0)\end{array}$ & $\begin{array}{r}2121,3^{a} \\
(2566,0)\end{array}$ \\
\hline
\end{tabular}

Les types de parcelles portant la même lettre ( $\mathrm{a}$ ou b) sur la même colonne ne sont pas significativement différents au seuil de 5 \%

(Test LSD de Fischer) pour la classe de hauteur considérée. Ceux portant les lettres ab sont statistiquement intermédiaires entre le groupe des « a » et ceux des « b » sur la même colonne. Les écart-types sont indiqués entre parenthèses. 


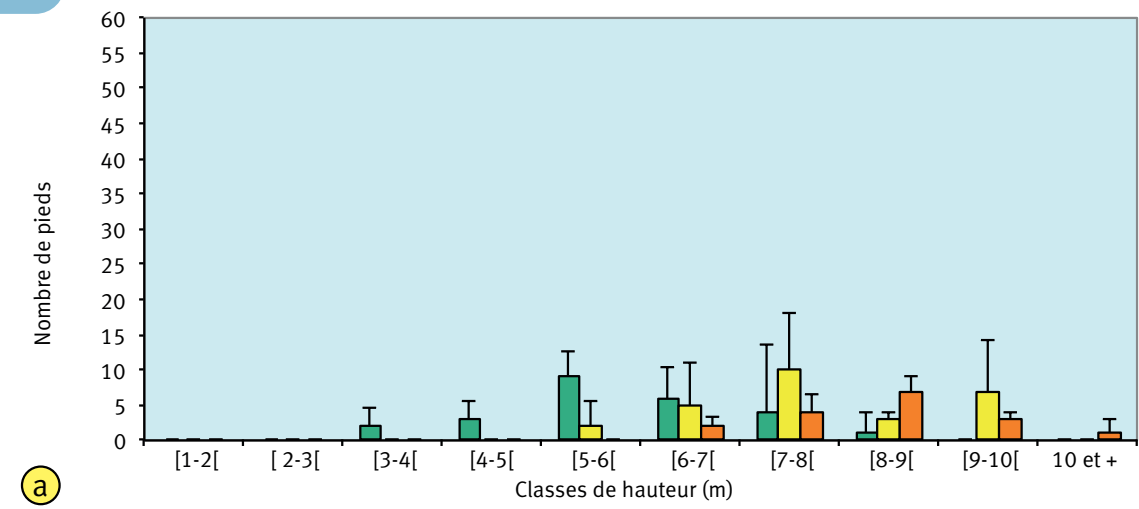

口Jeunes champs (2 ans) $\square$ Champs d'âge intermédiaire (6 ans) पVieux champs (plus de 15 ans)

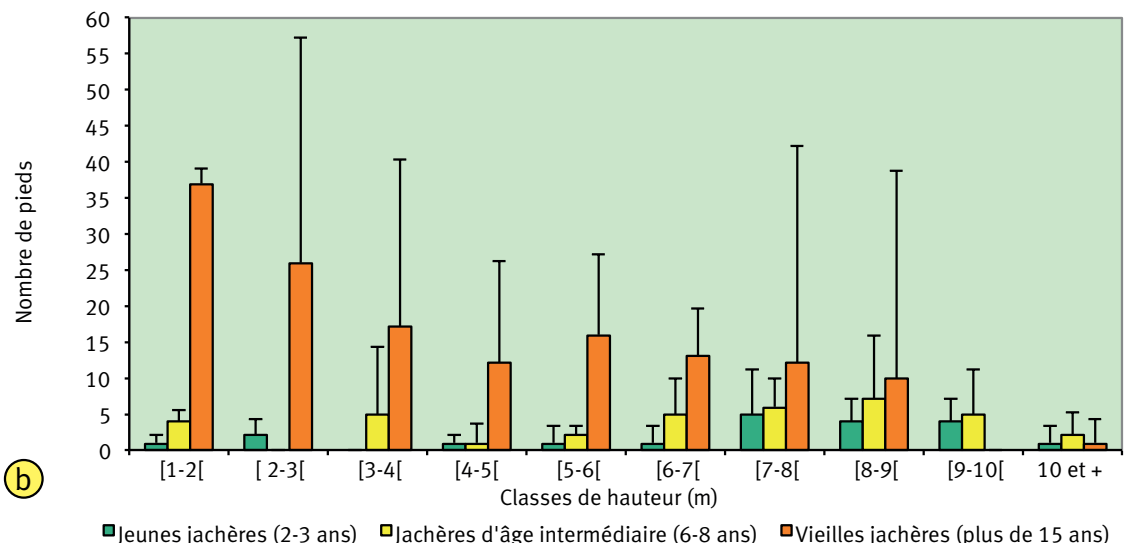

口jeunes jachères (2-3 ans) प 口Jachères d'âge intermédiaire (6-8 ans) पVieilles jachères (plus de 15 ans)

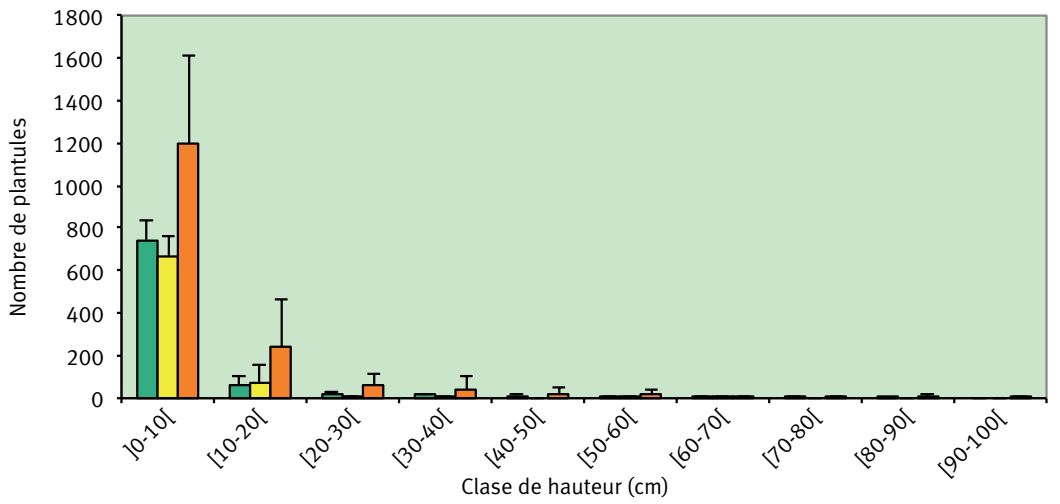

口jeunes jachères (2-3 ans) पajachères d'âge intermédiaire (6-8 ans) पVieilles jachères (plus de 15 ans)

Figure 1.

Structure verticale des karités adultes (a : champs ;

$\mathrm{b}$ : jachères) et de la régénération (c).

Contrairement aux champs, dans les jachères sont notés des individus dans pratiquement toutes les classes de hauteur supérieures ou égales à un mètre (photo 5). Le nombre de pieds augmente avec l'âge des jachères, plus nettement dans les petites classes que dans les classes suivantes (figure $1 \mathrm{~b}$ ). Dans toutes les jachères, la régénération est marquée par une majorité de plantules de moins de
$20 \mathrm{~cm}$ de hauteur. Après la classe de moins de $10 \mathrm{~cm}$, le nombre de plantules entre 10 et $20 \mathrm{~cm}$ subit une brusque diminution et se réduit sévèrement dans les classes qui suivent (figure 1c).

\section{Répartition spatiale}

Dans les champs, la distribution spatiale des karités de plus de $4 \mathrm{~m}$ de hauteur montre des pieds irrégulièrement répartis avec des écartements qui varient de $1 \mathrm{~m}$ à plus de $10 \mathrm{~m}$. La plupart des rares plantules recensées se trouvent sous le houppier des karités, à l'exception de quelques-unes dispersées ailleurs dans les champs (figures $2 \mathrm{a}, 2 \mathrm{~b}, 2 \mathrm{c}$ ).

Au sein des jeunes jachères et des jachères d'âge intermédiaire, une similitude est constatée avec les champs pour ce qui est de la distribution des karités de plus de $4 \mathrm{~m}$ de hauteur. Par contre, la distance entre ces individus est réduite dans les vieilles jachères. La régénération se regroupe autour des adultes (photo 6). Néanmoins, certaines plantules sont à l'écart des adultes. Il existe également des adultes sous lesquels ne pousse aucune plantule. Dans les vieilles jachères, les jeunes pieds de 1 à $4 \mathrm{~m}$ de hauteur se développent sous les grands karités ou à quelques mètres d'eux (figures 2 d, 2 e, 2 f).

\section{Densités à l'hectare}

La densité (ou fréquence) des tiges d'au moins $4 \mathrm{~m}$ de hauteur chute d'une dizaine de pieds à l'hectare entre les jeunes champs (31 pieds/ha) et les vieux (23 pieds/ha), alors qu'elle s'accroît nettement entre les jeunes jachères (23 pieds/ha) et les vieilles (85 pieds/ha) (tableau II). Néanmoins, la différence de densité n'est significative qu'entre les vieilles jachères et l'ensemble des autres types de parcelles $(P=0,034 ; R=79,0 \%)$. Les sujets ayant entre 1 et $4 \mathrm{~m}$ de hauteur sont faiblement représentés dans les jeunes champs (moins de trois à l'hectare) et sont totalement absents dans les autres types de champs. Leur fréquence est significativement plus importante dans les vieilles jachères (109 pieds/ha) que dans les autres parcelles ( $R=77,4 ; P=0,047 \%)$. Les densités de jeunes brins de moins d'un mètre de hauteur ne sont pas significativement différentes entre les parcelles (tableau III). La plus faible et la plus forte densité de plantules se retrouvent respectivement dans les vieux champs (210 plantules/ha) et dans les vieilles jachères (2 120 plantules/ha). 

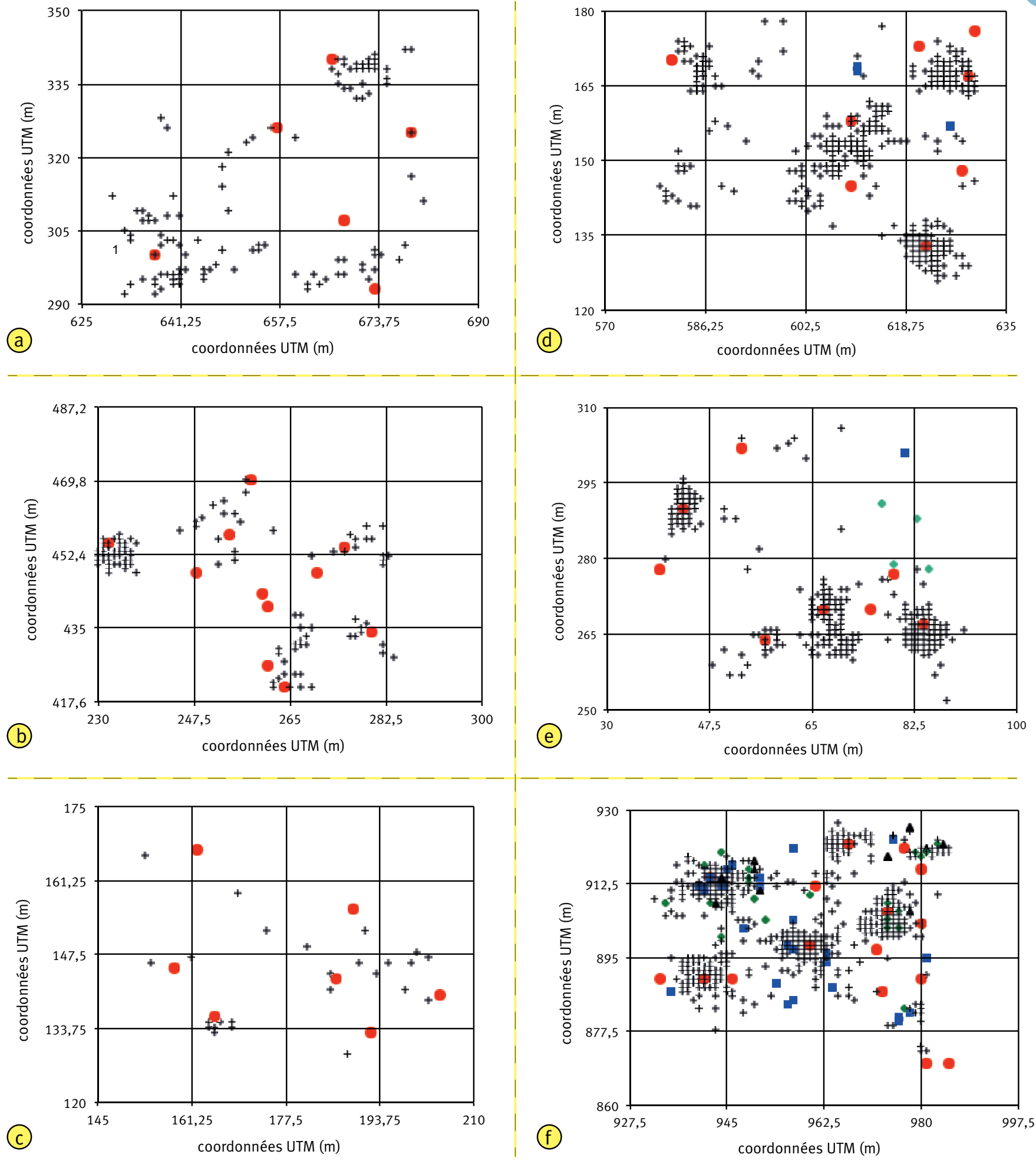

$+] 0-0,5 \mathrm{ml} ; \quad \bullet[0,5-1 \mathrm{ml} ; \quad \bullet[1-1,5 \mathrm{~m}[; \quad \mathbf{Q}[1,5-4 \mathrm{~m}[; \quad \bigcirc \geq 4 \mathrm{~m}$

Figure 2.

Distribution spatiale des karités dans un jeune champ (a),

un champ d'âge intermédiaire (b), un vieux champ (c),

une jeune jachère (d), une jachère d'âge intermédiaire (e)

et une vieille jachère (f). 


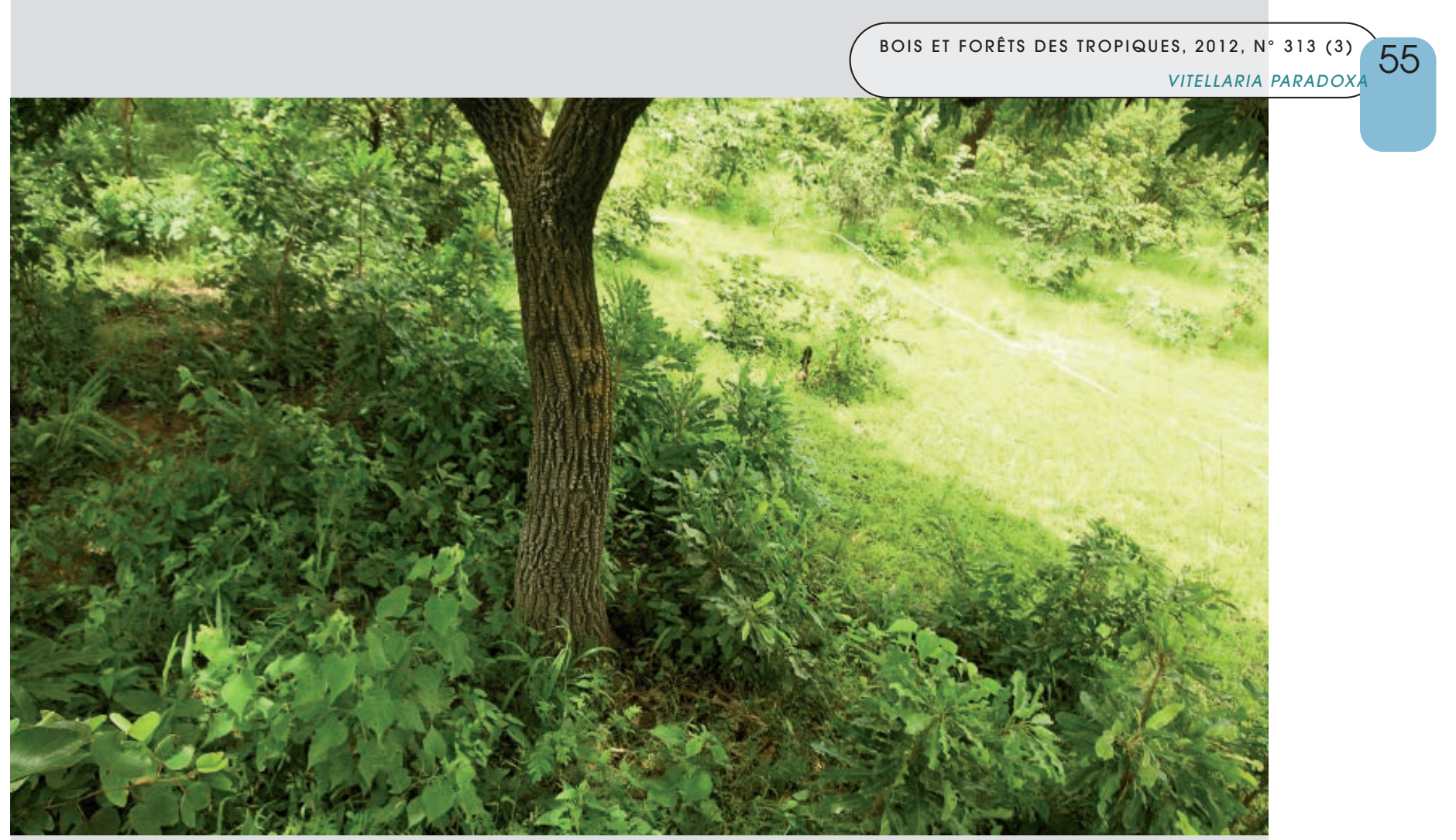

Photo 6.

Importante régénération sous un karité dans une jeune jachère de 3 ans.

Photo S. A. Kaboré.

\section{Pratiques agricoles}

Il ressort de l'enquête que, sur le terroir de Sobaka, les champs sont cultivés en continu en moyenne depuis 12 ans. Les plus vieux le sont depuis plus de 25 ans. Les terrains abandonnés en jachères le sont depuis 10 ans en moyenne ; $65 \%$ des enquêtés disent ne plus pratiquer l'alternance champjachère par manque de terres cultivables. Tous les enquêtés utilisent la charrue (culture attelée) et la daba comme outils de travail. Aucune protection particulière n'est accordée aux pieds des karités en dehors de la préservation contre le feu citée par seulement un tiers des enquêtés ; $40 \%$ des individus affirment épargner les plantules de karité lors des travaux champêtres (photo 7) et $60 \%$ disent ne pas le faire pour des difficultés de protection des plantules durant les labours.

\section{Discussion}

Il convient d'abord de préciser que le karité est une espèce protégée au Burkina Faso et donc interdite de coupe. Il fait partie des espèces prioritaires en matière de recherche scientifique. Il est également l'espèce préférée des paysans du fait de l'importance des ressources financières générées par la vente de ses amandes. Les karités dans les champs appartiennent au propriétaire de la parcelle qui l'exploite à sa guise. L'étude menée sur cette espèce à Sobaka montre des différences nettes dans la structure des populations en fonction de l'âge des champs et des jachères. Ces différences portent à la fois sur la structure verticale des populations, sur leur densité et sur la répartition spatiale des individus.

\section{Différences dans la structure verticale des populations}

En considérant la structure verticale des populations de karités dans les différents types de peuplements, le classement suivant est obtenu en allant des structures déséquilibrées aux structures les plus stables : vieux champs (plus de 15 ans), champs d'âge intermédiaire (6 ans), jeunes champs ( 2 ans), jeunes jachères (2 à 3 ans), jachères d'âge intermédiaire (6 à 8 ans) et vieilles jachères (plus de 15 ans). Toutefois, les parcs ici étudiés sont relativement plus jeunes que ceux du Nord du pays où la sénescence des arbres est encore plus alarmante (BonKoungou, 1987). L'absence de renouvellement des adultes fait communément penser que la régénération est absente des champs, et pourtant des plantules apparaissent dans les champs même si elles ne s'y épanouissent pas en jeunes pieds. La raison en est que ces plantules sont continuellement arrachées lors des opérations de culture. En outre, la lenteur de leur croissance n'encourage pas les paysans à les conserver et à les laisser grandir. Il s'ensuit, en accord avec DjossA et al. (2008), que la réduction voire l'absence des jeunes adultes compromet dangereusement la viabilité des parcs. Les individus d'un à trois mètres de hauteur, absents des champs, progressent dans les jeunes jachères et ce rééquilibrage de la structure des karités par l'apport de tels jeunes pieds est l'un des résultats les plus importants offerts par la mise en jachère prolongée. Un faible nombre d'individus s'affranchissent du stade plantule pour passer au stade adulte. La relative lenteur de la croissance des jeunes plants, qui se situe entre 18 et 35 $\mathrm{cm} /$ an pour la hauteur et entre 1,30 et $2,10 \mathrm{~cm} /$ an pour le diamètre de la tige selon la provenance des graines (BAYALA et al., 2009), explique en partie cette situation. En outre, l'abondante régénération au stade de semis subit de très fortes perturbations (feux de brousse, etc.) induisant l'abscission naturelle au cours de la saison sèche malgré un système racinaire assez profond. 

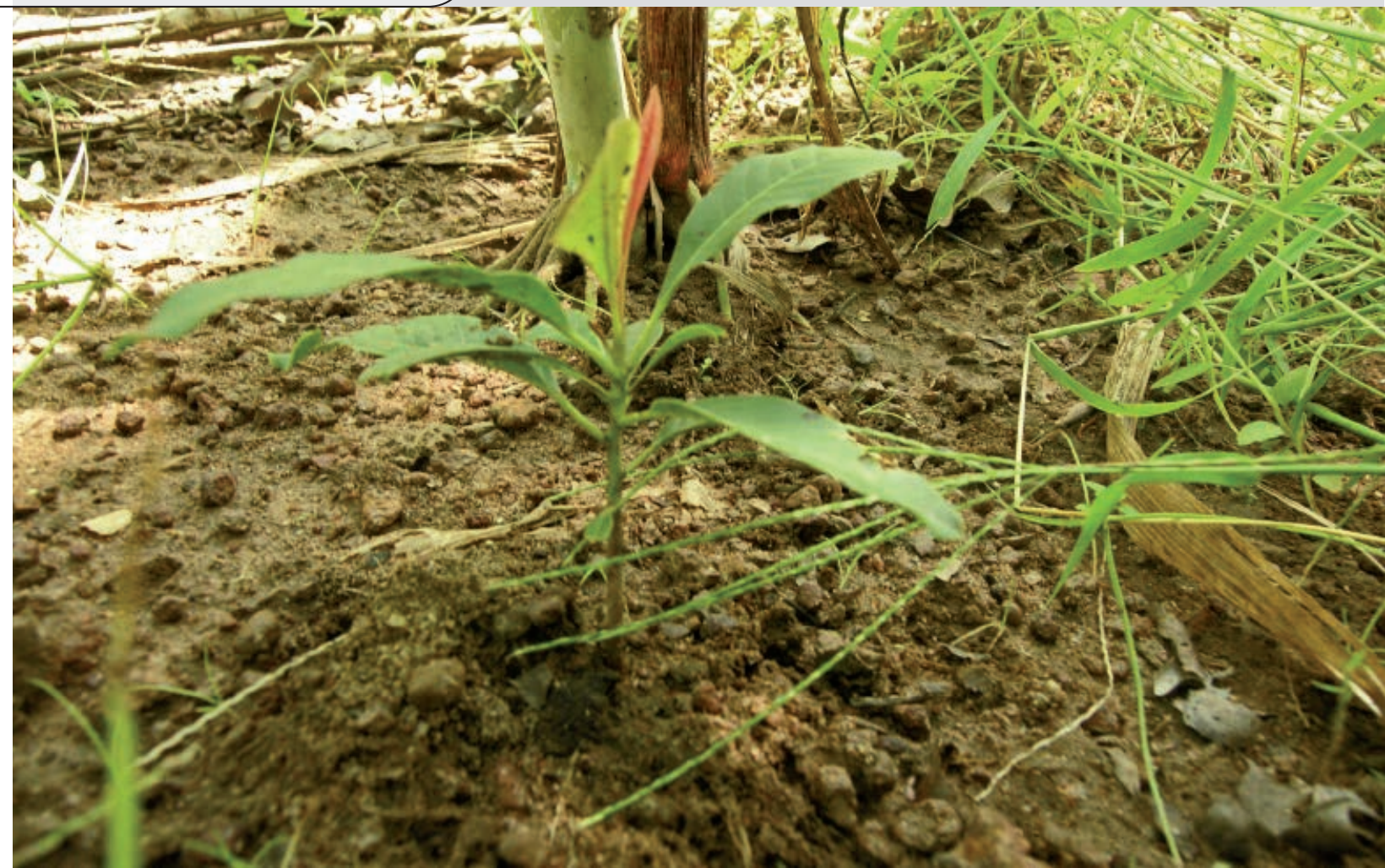

Photo 7.

Plantule de karité épargnée pendant les sarclages dans un champ.

Photo S. A. Kaboré.

\section{Différences dans la répartition spatiale des karités}

En champ, la répartition assez aléatoire des karités adultes semble témoigner du fait que le paysan ne s'intéresse ni à l'arrangement, ni à la densité des arbres qu'il entretient. Ce qui laisse à penser que l'arbre est une bonne opportunité que le paysan sait mettre à profit, sans pour autant agir en vue de la construction et de la gestion du parc. Les arbres se répartissent assez régulièrement dès que la densité devient relativement faible, mode de distribution qui convient mieux aux paysans car il homogénéise la lumière et l'ombrage au bénéfice des cultures (KELLY et al., 2004).

En jachère, le mode de distribution des différents individus de karité se précise avec l'âge des parcelles. Les juvéniles sont le plus souvent agrégés autour des grands karités. Ce résultat trouve son explication dans la barochorie de l'espèce. C'est pourquoi Kelly (2005) parle d'« attraction » des petits pieds de karité par les gros et DjossA et al. (2008) utilisent le terme d' " association » entre plantules et adultes. Le recrutement des jeunes pieds se fait de manière centrifuge du houppier du semencier vers l'espace environnant. L'ombrage de l'arbre mère ne semble pas nuire au bon développement des plantules qui y trouvent en revanche une plus grande humidité après les pluies et une meilleure protection contre les fortes températures au cours de la saison sèche. De manière générale, la distribution des pieds tend à être de plus en plus agrégée en passant du champ aux jachères d'âge avancé (KELLY, 2005).

\section{Différences dans la densité des populations}

La fréquence d'adultes diminue en fonction de l'âge des champs. Le karité connaît donc une dynamique régressive dans le terroir. Aucune étude n'a été menée dans le terroir pouvant permettre de comparer les densités passées et présentes, néanmoins, selon des personnes bénéficiant du recul dans le temps, le parc à karités a baissé en densité et continue à reculer. Du fait du statut et de l'importance du karité, les paysans se gardent d'abattre les vieux karités même improductifs qui finissent par mourir de mort naturelle. Les densités trouvées restent similaires à celles de KELLY et al. (2004) au Mali (16 et 31 karités par hectare respectivement à Mperesso et à Koumantou). À l'opposé, les densités moyennes des adultes augmentent avec l'âge des jachères. Les vieilles jachères offrent un nombre important de plants (2 121 pieds/ha de moins d'un mètre et 109 pieds/ha pour la classe 1 à $4 \mathrm{~m}$ ) pouvant être sélectionnés suivant différentes classes de hauteur et être protégés lors de la remise en culture. L'absence de différence significative entre les parcelles en ce qui concerne la densité des plantules indique qu'elles subissent toutes les effets de divers facteurs qui les perturbent fortement, tels que le labour, les feux et les longues périodes de sécheresse allant de novembre à mai. 


\section{Prise en compte des autres ligneux dans la dynamique du karité}

En intégrant les autres ligneux dans l'analyse de la dynamique du karité, il apparaît que les longues jachères permettent la recolonisation de plusieurs espèces. Il a été possible de remarquer en particulier le retour (du champ à la jachère) d'un grand nombre d'espèces comme Piliostigma thonningii, Detarium microcarpum et Combretum glutinosum. Ces résultats sont similaires à ceux de KELlY (2005) sur la remarquable présence de Piliostigma thonningii à côté des karités. La recherche d'un rajeunissement harmonieux des parcs à karités ne peut se faire sans tenir compte des autres espèces utilitaires devant aussi être préservées. Malencontreusement, ces essences telles que Parkia biglobosa, Tamarindus indica, Lannea microcarpa ou Bombax costatum sont peu représentées en tant qu'adultes, avec une très faible régénération au sein des parcs et des jachères ; leurs dynamiques sont régressives. Pourtant certaines pratiques agricoles que sont la protection des plantules lors des travaux agricoles, la régénération naturelle assistée (Rna) ou la mise en jachère de longue durée sont bien connues pour avoir un impact favorable sur la dynamique des espèces ligneuses. L'enquête a permis de faire l'état du niveau d'adoption de ces pratiques sur le terroir de Sobaka et d'en évaluer les conséquences sur la dynamique de Vitellaria paradoxa dans l'espace agraire.

\section{Absence de protection des plantules lors des travaux champêtres et de régénération}

L'enquête sur les pratiques agricoles montre que la majorité des agriculteurs n'épargnent pas les plantules de karité lors des travaux en champ. Les conséquences de cette pratique vis-à-vis de la dynamique de Vitellaria paradoxa sont évidentes : les pieds qui meurent ne sont pas remplacés, induisant inexorablement le vieillissement des populations de karités. Pour espérer maintenir ou augmenter la production fruitière, le paysan décidant d'entretenir dans son parc de jeunes individus doit tenir compte du fait que cette production fruitière démarre autour de la vingtième année et que la pleine production ne débute qu'à 40 ans (Bonkoungou, 1987). Il s'agit donc d'une activité dont les bénéfices sont attendus à long terme, ce qui peut constituer un motif de découragement. Le karité peut vivre jusqu'à 200 ans mais son rendement en fruits est alors très amoindri (BONKOUNGOU, 1987). L'inadéquation entre labour et protection des plantules est systématiquement évoquée par les paysans pour justifier les difficultés d'épargner quelques jeunes pieds. Les outils aratoires (charrue, daba) et les types de cultures adoptés (céréales principalement) ne sont pas dans la pratique un handicap majeur, pour protéger quelques plantules de karité. En plus, les surfaces occupées par les karités ne représentent qu'à peine $11 \%$ des vieux champs. Il existe un débat contradictoire sur l'effet de l'ombrage des arbres sur les différentes productions agricoles. En fait, c'est davantage la volonté du paysan qui prime quant à la convenance de sauvegarder les jeunes individus. L'absence de protection ou de plantation de jeunes pieds de karité dans les champs peut s'expliquer en tenant compte de l'héritage culturel et technique des agriculteurs. Par le passé, les pieds sélectionnés et protégés procuraient des fruits en suffisance et leur nature initiale devait bien convenir aux besoins. Toutefois, la longévité du karité a pu faire en sorte que les paysans ne se sont, hélas, pas réellement rendu compte du déclin et du vieillissement progressifs des peuplements et que de ce fait ils n'ont pas été à même d'adopter en leur temps les mesures idoines qui s’imposaient.

\section{Réduction de la durée de mise en jachère}

Les résultats ont montré que la jachère est la méthode par excellence pour le rajeunissement naturel de la population de karités. Cependant, sur le terroir de Sobaka, faute d'espace suffisant, les parcelles cultivables ne sont plus disponibles, ce qui n'autorise plus l'abandon des champs au profit de la jachère. La moyenne d’âge des jachères encore existantes est de dix ans, alors qu'un laps de temps de plus de quinze années est nécessaire pour un bon rajeunissement naturel des karités. Une vingtaine d'années auparavant, Somé (1992) rapportait que, de l'avis du paysan, il lui serait difficile de concevoir son terroir autrement que sans la jachère qui a toujours eu sa place dans l'organisation et la structuration de Sobaka. Ce changement est aussi vécu ailleurs dans le pays où AugussEAu et al. (2006) et RABILD et al. (2011) ont clairement relevé le raccourcissement de l'âge des jachères à moins de quinze ans, voire leur abandon pur et simple. Pour prendre en main la situation, étant donné que l'essentiel de la régénération se fait sous le houppier des karités, le paysan devra veiller, lors de la remise en culture des jachères, à bien sélectionner les jeunes sujets situés assez loin des vieux pieds s’il juge nécessaire de conserver ces derniers. Par ailleurs, l'intensification et la mécanisation agricole, entraînant des dégâts irrémédiables, sont de nouveaux défis pour le maintien des densités du parc à karités (DIARRASSOUBA et al., 2009 ; ASSÉ, LASSOIE, 2011). Enfin, YELEMou et al. (2007) rapportent également le danger de voir des espèces inhabituelles des parcs agroforestiers comme Piliostigma reticulatum prendre de plus en plus la place des espèces agroforestières traditionnelles en manque de régénération dans le système agraire du Plateau central burkinabé. 


\section{Conclusion}

Les résultats de la présente étude permettent d'avancer que la dynamique du karité, Vitellaria paradoxa, est bien liée aux durées respectives des champs et des jachères et que le type d'utilisation des terres l'influence incontestablement. Certes, l'espèce prolifère abondamment, mais, du fait des pratiques agricoles courantes, les plantules rencontrent de nombreux obstacles pour se développer dans les champs. L'étude met en évidence l'importance des jachères de longue durée pour mener à bien la restauration des peuplements naturels de karité. Sous des conditions écologiques semblables à celles de Sobaka, les jachères devraient atteindre une durée minimale de quinze années pour être efficaces. Dans les zones où la pratique de la jachère de longue durée est pratiquement impossible du fait de la pression foncière, plusieurs techniques de régénération sont envisageables pour enrichir les parcs à karités et assurer le renouvellement de la ressource :

- en premier, il s'agit de mettre à profit l'exploitation de la régénération spontanée en protégeant des plantules en nombre suffisant, bien réparties et positionnées dans les champs en vue de remplacer à moyen terme les arbres vieillissants ;

- autrement, sachant que le karité en plantation donne des résultats probants, un plan de rajeunissement par reboisement peut être adopté en vue de densifier les peuplements de karités et de remplacer progressivement les pieds sénescents ;

- enfin, on peut recourir à la régénération naturelle assistée qui consiste à accompagner et favoriser le développement des jeunes plants en les taillant, en les dégageant, voire en leur apportant des nutriments. Mais reste encore à mener une étude concrète sur les différentes méthodes de régénération en vue de proposer des techniques pratiques adaptées à chaque zone écologique de l'aire de distribution du karité, qui soient appropriables par les agriculteurs, et contribuer ainsi à la domestication de l'espèce.

Quoi qu'il en soit, l'essentiel repose sur une meilleure sensibilisation du monde rural vis-à-vis de l'avenir des parcs agroforestiers et des conséquences défavorables qui peuvent résulter de la pression foncière.

\section{Remerciements}

Les auteurs souhaitent remercier le projet INNOVKAR (Innovative Tools and Techniques for Sustainable Use of the Shea Tree in Sudano-Sahelian zone) pour avoir financé les travaux d'étude. Les remerciements s'adressent aussi aux paysans de Sobaka pour leur collaboration. Les auteurs sont particulièrement reconnaissants envers Daniel Kibora, Abel Kaboré, Madi Kagambèga, Amidou Zoundi et feu Bobo Koulibaly pour leur aide sur le terrain.
ASSÉ R., LASSOIE J. P., 2011. Household decision-making in agroforestry parklands of Sudano-Sahelian Mali. Agroforestry Systems, 82 (3): 247-261. Doi 10.1007/s10457011-9395-2.

AUGUSSEAU X., NIKIÉMA P., TORQUEBIAU E., 2006. Tree biodiversity, land dynamics and farmers' strategies on the agricultural frontier of southwestern Burkina Faso. Biodiversity and Conservation, 15 (2): 613-630. Doi 10.1007/s10531005-2090-8.

BAYALA J., OUEDRAOGO S. J., TEKLEHAIMANOT Z., 2008. Rejuvenating indigenous trees in agroforestry parkland systems for better fruit production using crown pruning. Agroforestry Systems, 72 (3): 187-194. Doi 10.1007/s10457007-9099-9.

BAYALA J., OUEDRAOGO S. J., ONG K., 2009. Early growth performance and water use of planted West African provenance of Vitellaria paradoxa C.F. Gaertn. (karité) in Gonsé, Burkina Faso. Agroforestry Systems, 75 (2): 117-127. Doi 10.1007/s10457-008-9167-9.

BONKOUNGOU E. G., 1987. Monographie du Karité (Butyrospermum paradoxum (Gaertn. f. Hepper), espèce agroforestière à usages multiples. Ouagadougou, Burkina Faso, Irbet/Cnrst, $67 \mathrm{p}$.

CHEVALIER A., 1946. L'arbre à beurre d'Afrique et l'avenir de sa culture. Oléagineux, 1 : 7-11.

DIARRASSOUBA N., LFOFANA J., BAKAYOKO A., NGUESSAN A. K., SANGARE A., 2009. Influence des systèmes agraires sur la dynamique de régénération naturelle du karité : Vitellaria paradoxa C.F. Gaertn. (Sapotaceae) en Côte D’Ivoire. Agronomie Africaine, 21 (1). ISSN: 1015-2288. Site Web : www.ajol.info (consulté le 31 Juillet 2012).

DJOSSA B. A., FAHR J., WIEGAND T., AYIHOUENOU B. E., KALKO E. K., SINSIN B. A., 2008. Land use impact on Vitellaria paradoxa C.F. Gaertn. stand structure and distribution patterns: a comparison of Biosphere Reserve of Pendjari in Atacora district in Benin. Agroforestry Systems, 72 (3): 205 220. Doi 10.1007/s10457-007-9097-y.

GWALI S., OKULLO J. B. L., EILU G., NAKABONGE G., NYEKO P., VUZI P., 2012. Traditional management and conservation of shea trees (Vitellaria paradoxa subspecies nilotica) in Uganda. Environment, Development and Sustainability, 14 (3): 347-363. Doi 10.1007/s10668-011-9329-1.

HALL J. B., AEBISCHER D. P., THOMLINSON H. F., OSEI-AMANING E., HINDLE J. R., 1996. Vitellaria paradoxa a monograph. Bangor, Royaume-Uni, School of Agriculture and Forest Sciences, University of Wales, $105 \mathrm{p}$.

INSD, 2009. Annuaire statistique 2008. Ouagadougou, Burkina Faso, Institut national de la statistique et de la démographie, 413 p. Site Web : www.insd.bf (consulté le 30 juillet 2012). 
KELLY B. A., BOUVET J.-M., PICARD N., 2004. Size class distribution and spatial pattern of Vitellaria paradoxa in relation to farmers' practices in Mali. Agroforestry Systems, 60 (1): 3-11. KELLY B. A., 2005. Impact des pratiques humaines sur la dynamique des populations et sur la diversité génétique de Vitellaria paradoxa Gaertn. (karité) dans les systèmes agroforestiers au sud du Mali. Thèse, Université de Bamako, Mali, $233 \mathrm{p}$.

KELLY B. A., GOURLET-FLEURY S., BOUVET J.-M., 2007. Impact of agroforestry practices on the flowering phenology of Vitellaria paradoxa in parklands in southern Mali. Agroforestry Systems, 71 (1): 67-75. Doi 10.1007/s10457-0079074-5.

LAFLEUR M., 2008. Recherches et documentation des meilleures pratiques pour la gestion durable des parcs à karités en Afrique de l'Ouest. Montréal (Québec), Canada, Centre d'étude et de coopération internationale, 110 p. Site Web : www.ceci.ca (consulté le 20 novembre 2011).

LAMIEN N., 2006. Fructification du karité (Vitellaria paradoxa C.F. Gaertn. Sapotaceae) : facteurs de déperdition, amélioration et prévision des rendements à Bondoukuy, Ouest Burkina Faso. Thèse de doctorat, Université de Ouagadougou, Burkina Faso, $101 \mathrm{p}$.

NIKIÉMA A., VAN DER MAESEN L. J. G., HALL J. B., 2003. The impact of parkland management practices on plant resources diversity. In: Teklehaimanot Z. (éd.). Improved management of agroforestry parkland systems in Sub-Saharan Africa. EU/INCO Project Contract IC18-CT98-0261. Third Annual Progress Report, Bangor, Royaume-Uni, University of Wales.

NOUVELLET Y., KASSAMBARA A., BESSE F., 2006. Le parc à karités au Mali : inventaire, volume, houppier et production fruitière. Bois et Forêts des Tropiques, 228 : 5-20.

PELTIER R., FORKONG C. N., NTOUPKA M., MANLAY R., HENRY M., MORILLON V., 2007. Évaluation du stock de carbone et de la productivité en bois d'un parc à karités du Nord-Cameroun. Bois et Forêts des Tropiques, 294 : 39-50.

RAEBILD A., LARSEN A. S., JENSEN J. S., OUEDRAOGO M., DE GROOTE S., VAN DAMME P., BAYALA J., DIALLO B. O., SANOU H., KALINGANIRE A., KJAER E. D., 2011. Advances in domestication of indigenous fruit trees in the West African Sahel. New Forests, 41 (3): 297-315. Doi 10.1007/s11056-010-9237-5.

RAEBILD A., HANSEN U. B., KAMBOU S., 2011. Regeneration of Vitellaria paradoxa and Parkia biglobosa in a parkland in southern Burkina Faso. Agroforestry Systems, 85 (3): 443453. Doi 10.1007/s10457-011-9397-0.

SANOU H., KAMBOU S., TEKLEHAIMANOT Z., DEMBÉLÉ M., YOSSI H., SINA S., DJINGDIA L., BOUVET J.-M., 2004. Vegetative propagation of Vitellaria paradoxa by grafting. Agroforestry Systems, 60 (1): 93-99.
SERPENTIÉ G., BAYALA J., HELMFRID S., LAMIEN N., 1996. Pratiques et enjeux de la culture du karité (Butyrospermum paradoxum Gaertn. F. Hepper) dans l'Ouest du Burkina Faso. In : Floret C. (éd.). La jachère, lieu de production. Actes de l'atelier Coraf/Orstom/Union européenne, 2-4 octobre 1996, Bobo-Dioulasso, Burkina Faso. Dakar, Sénégal, Orstom, 59-72.

SOMÉ N. A., 1992. Conception et pratique de la jachère dans le terroir de Sobaka (Burkina Faso). Mémoire de diplôme d'études supérieures universitaires de biologie végétale tropicale, Université Pierre et Marie Curie, France, $44 \mathrm{p}$.

SOMÉ N. A., 1996. Les systèmes écologiques post-culturaux de la zone soudanienne (Burkina Faso). Structure spatiotemporelle des communautés végétales et évolution des caractères pédologiques. Thèse, Université Pierre et Marie Curie, France, 212 p.

YELEMOU B., BATIONO B. A., YAMEOGO G., MILLOGO-RASOLODIMBY J., 2007. Gestion traditionnelle et usages de Piliostigma reticulatum sur le Plateau central du Burkina Faso. Bois et Forêts des Tropiques, 291 : 55-66. 